\title{
A Cyclotomic Lattice Based Closed Loop QOSTBC for Four Transmit Antennas
}

\author{
K. Senthil Kumar, M. Palanivelan, M. Sivaram, P. Shanmugapriya,V. Bakyalakshmi
}

\begin{abstract}
Drawback of the Space Time Block Code (STBC) is, for complex constellations, full rate and full diversity design exist only for two transmit antennas. In this paper, we propose a novel closed loop Quasi Orthogonal Space Time Block Code (QOSTBC) system based on cyclotomic lattices for four transmit antennas. It is a full rate and full diversity design where the information bits are mapped into four dimensional (4-D) lattice points. The bit error and symbol error performance of the system are evaluated by simulation.
\end{abstract}

Index Terms: MIMO, QOSTBC, cyclotomic, lattice, diversity product.

\section{INTRODUCTION}

$4 \mathrm{G}$ and $5 \mathrm{G}$ are required to have improved quality and data rate services. But, the time varying multipath fading makes reliable wireless transmission as a difficult task. Various techniques like transmitter power control, Time diversity [1], Frequency diversity [2], Time interleaving have been adopted to overcome fading. A practical, more effective and widely adopted technique is antenna diversity [3] and [4]. Much attention is paid on transmitter diversity which involves many antennas for transmission. Space Time Trellis Coding (STTC) is appropriate to multiple transmit antennas[5][6][7] and [8]. However, when the number of transmit antennas is fixed, the decoding complexity of STTC increases exponentially with transmission rate.

A simple Transmit diversity was proposed by Alamouti [9] in 1998, regarded as the first Orthogonal Space Time Block Code (OSTBC). Because of its simplicity, STBCs became an attractive approach for transmit diversity. However, for complex constellations, the code rate falls from unity [10]-[15]. The diversity order can be increased by choosing signals from rotated constellations [16], [17]. A closed loop scenario is presented in [18] which increases the transmit diversity by feeding back channel information using one bit. A new QOSTBC design based on cyclotomic lattice was proposed in [19], which is an open loop system. The contribution of the proposed scheme is conversion of the open loop system proposed in [19] into a closed loop system based on the scheme proposed in [18].

Revised Version Manuscript Received on Jun 20, 2019.

Dr. M. Palanivelan, Professor \& Head, Department of ECE, Rajalakshmi Engineering College, Chennai.

K. Senthil Kumar, Associate Professor, Department of ECE, Rajalakshmi Engineering College, India.

V. Bakyalakshmi, Assistant Professor, Department of ECE, Rajalakshmi Engineering College, India. Her research interests include wireless communication and optical.

\section{SYSTEM MODEL}

The codeword S [20] is given by Eq (1)

$$
\mathrm{S}_{\mathrm{J}}=\left[\begin{array}{cccc}
s_{1} & s_{2} & s_{3} & s_{4} \\
-s_{2}^{*} & s_{1}^{*} & -s_{4}^{*} & s_{3}^{*} \\
-s_{3}^{*} & -s_{4}^{*} & s_{1}^{*} & s_{2}^{*} \\
s_{4} & -s_{3} & -s_{2} & s_{1}
\end{array}\right]
$$

The received signal is given by

$$
\mathrm{R}=\mathrm{HS}_{\mathrm{J}}+\mathrm{N}
$$

where $\mathrm{H}$ represents the channel matrix and $\mathrm{N}$ represents the noise.

\section{LATTICE QOSTBC}

A four dimensional complex cyclotomic lattice $\Gamma_{4}\left(\mathrm{G}_{4 \times 4}\right)$ [19] and [21] over a ring of integers $Z\left[\zeta_{\mathrm{m}}\right]$ is given by

$$
\Gamma_{4}\left(\mathrm{G}_{4 \times 4}\right)=\left\{\mathbf{v}=\mathrm{G}_{4 \times 4} \mathrm{a} \mid \mathrm{a} \in \mathrm{Z}\left[\zeta_{\mathrm{m}}\right]^{4}\right\}
$$

where $\mathbf{v}=\left[\begin{array}{llll}v_{1} & v_{2} & v_{3} & v_{4}\end{array}\right]^{T}, a=\left[\begin{array}{llll}a_{1} & a_{2} & a_{3} & a_{4}\end{array}\right]^{T}, G_{4 \times 4}$ is a $4 \times 1$ complex matrix called the generating matrix, $\mathrm{v}$ is a point in the complex lattice, $a$ is the point in the real lattice, $m$ is a positive integer; $Z\left[\zeta_{\mathrm{m}}\right]=\left\{\mathrm{Z} \mid \mathrm{Z}=\mathrm{Z}_{1}+\mathrm{Z}_{2} \zeta_{\mathrm{m}}, \zeta_{\mathrm{m}}=\mathrm{e}^{\mathrm{j} 2 \pi / \mathrm{m}}, \mathrm{Z} 1, \mathrm{Z} 2\right.$ $\in \omega Z$; ; The Minimum Product Distance (MPD) of the $\Gamma_{4}\left(\mathrm{G}_{4 \times 4}\right)$ is defined as

$$
d_{M P D}=\min _{v \neq v^{\prime} \in \Gamma_{4}(G)}\left(\left|\Delta v_{1}\right|\left|\Delta v_{2}\right|\left|\Delta v_{3}\right|\left|\Delta v_{4}\right|\right)
$$

where $\mathrm{v}, \mathrm{v}^{\prime} \in \Gamma_{4}\left(\mathrm{G}_{4 \times 4}\right), \Delta v=\mathrm{v}-\mathrm{v}^{\prime}=\left[\Delta v_{1} \ldots \Delta v_{4}\right]^{\mathrm{T}}$ and $v \neq v^{\prime}$. The Lattice considered in this paper is a full diversity lattice i.e. $d_{M P D}>0$.

The block diagram of the proposed system is shown in the fig.1. Four lattice points $s_{1}, s_{2}, s_{3}$ and $s_{4}$ that are closer to the zero in the lattice have been selected. The incoming bit stream is mapped to these lattice points. After multiplying the entries of $\mathrm{S}_{\mathrm{J}}$ given by Eq.(1) by four phase factors, the proposed scheme is presented in Eq.(5).

The codeword $\mathrm{Sp}$ is given by

$$
\mathrm{S}_{\mathrm{p}}=\left[\begin{array}{cccc}
s_{1} e^{j \alpha} & s_{2} e^{j \beta} & s_{3} e^{j \gamma} & s_{4} e^{j \theta} \\
-s_{2}^{*} e^{-j \alpha} & s_{1}^{*} e^{-j \theta} & -s_{4}^{*} e^{-j \gamma} & s_{3}^{*} e^{-j \beta} \\
-s_{3}^{*} e^{-j \alpha} & -s_{4}^{*} e^{-j \beta} & s_{1}^{*} e^{-j \gamma} & s_{2}^{*} e^{-j \theta} \\
s_{4} e^{j \alpha} & -s_{3} e^{j \theta} & -s_{2} e^{j \gamma} & s_{1} e^{j \beta}
\end{array}\right]
$$

The received signals are given by

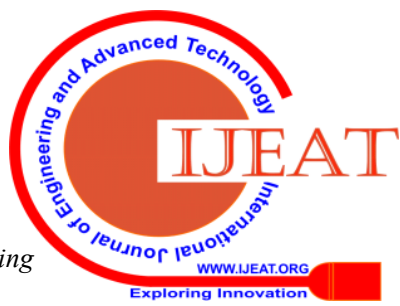


(8)

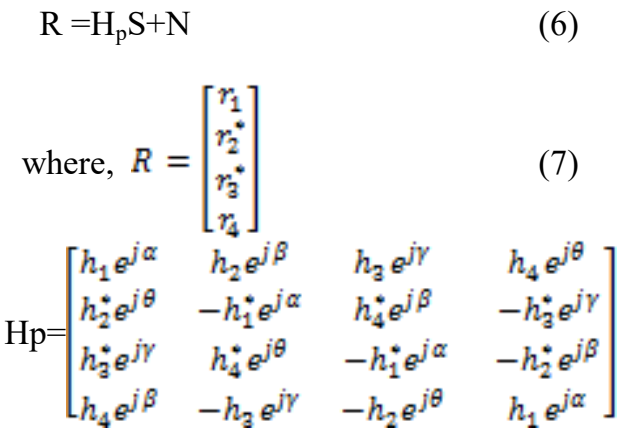

$$
S=\left[\begin{array}{l}
s_{1} \\
s_{2} \\
s_{3} \\
s_{4}
\end{array}\right] \text {,and } N=\left[\begin{array}{l}
n_{1} \\
n_{2}^{*} \\
n_{3}^{*} \\
n_{4}
\end{array}\right]
$$

The Grammian matrix $\mathrm{G}$ is calculated as

$$
G=H_{p}^{H} H_{p}=U_{p}+V_{p}
$$

where $U_{p}=h^{2}\left[\begin{array}{llll}1 & 0 & 0 & 0 \\ 0 & 1 & 0 & 0 \\ 0 & 0 & 1 & 0 \\ 0 & 0 & 0 & 1\end{array}\right]$

and $V_{p}=\omega\left[\begin{array}{cccc}0 & 0 & 0 & 1 \\ 0 & 0 & 1 & 0 \\ 0 & -1 & 0 & 0 \\ -1 & 0 & 0 & 0\end{array}\right]$

The parameter $\omega$ is given by Eq.(13)

$\omega=e^{j(\alpha-\beta)} \cdot 2 R e\left(h_{1}^{*} h_{4}\right)-e^{j(\gamma-\theta)} \cdot 2 R e\left(h_{2}^{*} h_{3}\right)$

(13)

To get full diversity, $\omega$ should be minimized. The $\omega$ value can be minimized by knowing the channel information. Based on the channel information, the factors $e^{j(\alpha-\beta)}$ and $e^{j(\gamma-\theta)}$ are adjusted. When $\operatorname{Re}\left(h_{1}^{*} h_{4}\right) \cdot \operatorname{Re}\left(h_{2}^{*} h_{3}\right) \geq 0$, the product $e^{j(\alpha-\beta)} \cdot e^{j(\gamma-\theta)}$ is set equal to 1 . Otherwise, the product is set equal to -1 .

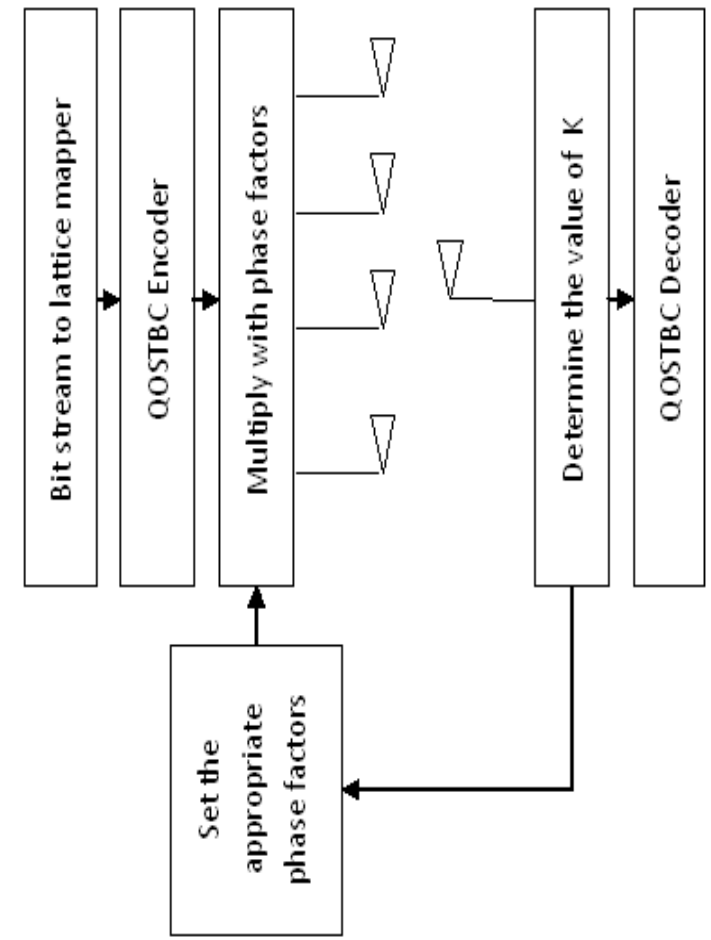

Fig. 1 Cyclotomic lattice based closed loop System

The parameter $\omega$ is given by Eq.(13)

$\omega=e^{j(\alpha-\beta)} \cdot 2 R e\left(h_{1}^{*} h_{4}\right)-e^{j(\gamma-\theta)} \cdot 2 R e\left(h_{2}^{*} h_{3}\right)$

To get full diversity, $\omega$ should be minimized. The $\omega$ value can be minimized by knowing the channel information. Based on the channel information, the factors $e^{j(\alpha-\beta)}$ and $e^{j(\gamma-\theta)}$ are adjusted. When $\operatorname{Re}\left(h_{1}^{*} h_{4}\right) \cdot R_{e}\left(h_{2}^{*} h_{3}\right) \geq 0$, the product $e^{j(\alpha-\beta)} \cdot e^{j(\gamma-\theta)}$ is set equal to 1 . Otherwise, the product is set equal to -1 .

The channel information is fed back to the transmitter using one bit. The feedback bit $\mathrm{k}$ is set equal to zero, when $R_{e}\left(h_{1}^{*} h_{4}\right) \cdot R_{e}\left(h_{2}^{*} h_{3}\right) \geq 0$. Otherwise, set $\mathrm{k}$ equal to one. On examining the value of $\mathrm{k}$, the phase factors are set as follows. The angles $\alpha$ and $\gamma$ in Eq.(5) are set equal to $180^{\circ}$, the angles $\beta$ and $\theta$ in Eq.(5) are set equal to zero, if $\mathrm{k}=1$. The angles $\alpha$, $\beta$ and $\theta$ are set equal to zero, the angle $\gamma$ is set equal to $180^{\circ}$, if $\mathrm{k}=0$. 


\section{SIMULATION RESULTS}

The parameters considered for simulating the proposed system is given in the Table I.

Table I Simulation parameters

\begin{tabular}{|l|l|}
\hline \multicolumn{1}{|c|}{ Channel } & Quasi-static, Flat fading \\
\hline Lattice points & S1 $=[-0.1399-0.1016 \mathrm{i}$ \\
& $0.5000+0.3633 \mathrm{i}$ \\
& $-0.0572-0.0416 \mathrm{i}$ \\
& $0.2045+0.1486 \mathrm{i}]$ \\
& $\mathrm{S} 2=-\mathrm{S} 1$ \\
& $\mathrm{~S} 3=[-0.3489+0.2605 \mathrm{i}$ \\
& $0.4563-0.0526 \mathrm{i}$ \\
& $0.2226+0.2692 \mathrm{i}$ \\
& $-0.2045+0.0616 \mathrm{i}]$ \\
& $\mathrm{S} 4=-\mathrm{S} 3$ \\
\hline Noise & AWGN \\
\hline Channel State & Known at both Transmitter \\
Information(CSI) & and Receiver end \\
\hline No. of Transmit & 4 \\
antennas & \\
\hline No. of Receive & 1 \\
antennas & \\
\hline Type of Decoding & Maximum Likelihood \\
& Decoding \\
\hline
\end{tabular}

As it is impossible to search the set of lattice points which are used here, $\Theta=\{-3,-1,1,3\}$ is chosen. The generating matrix $\mathrm{G}_{4 \times 4}$ considered in this paper is given by Eq.(14)

$$
\mathrm{G}_{4 \times 4}=\left[\begin{array}{cccc}
\lambda & \lambda^{2} & \lambda^{3} & \lambda^{4} \\
\lambda^{7} & \lambda^{14} & \lambda^{21} & \lambda^{28} \\
\lambda^{13} & \lambda^{26} & \lambda^{39} & \lambda^{52} \\
\lambda^{19} & \lambda^{38} & \lambda^{57} & \lambda^{76}
\end{array}\right]
$$

where $\lambda=e^{j \pi / 15}$ and the $\Gamma_{4}\left(\mathrm{G}_{4 \times 4}\right)$ is formed over a ring of integers $Z\left[\zeta_{6}\right][19][20]$ and [21].

Fig.3 compares the Bit error performance of cyclotomic lattice based open loop system and the proposed cyclotomic lattice based closed loop system.

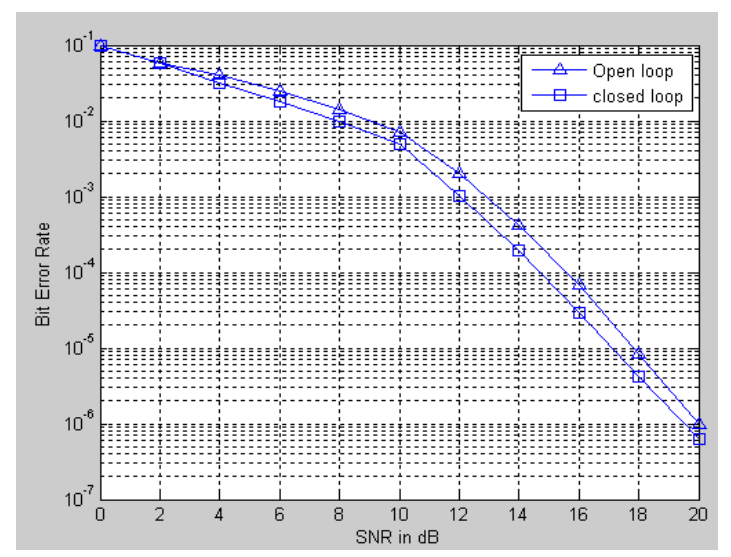

Fig. 3 Bit error rate versus Signal to noise ratio for lattice based open and closed loop QOSTBC

The proposed lattice based closed loop QOSTBC outperforms the open loop lattice based QOSTBC. Table II compares the Bit error rate of cyclotomic lattice based open loop system and the proposed cyclotomic lattice based closed loop system at different values of SNR. It is observed that at $12 \mathrm{~dB}$, the Bit error performance of the proposed closed loop system is $49.24 \%$ better than the open loop system.

Table II Signal to Noise Ratio Vs Bit Error Rate

\begin{tabular}{|l|l|l|}
\hline SNR in dB & $\begin{array}{l}\text { BER (Closed } \\
\text { Loop) }\end{array}$ & $\begin{array}{l}\text { BER (Open } \\
\text { Loop) }\end{array}$ \\
\hline 0 & $1.0 \times 10^{-1}$ & $1.0 \times 10^{-1}$ \\
\hline 2 & $5.7 \times 10^{-2}$ & $5.9 \times 10^{-2}$ \\
\hline 4 & $3.2 \times 10^{-2}$ & $4.1 \times 10^{-2}$ \\
\hline 6 & $1.8 \times 10^{-2}$ & $2.5 \times 10^{-2}$ \\
\hline 8 & $1.0 \times 10^{-2}$ & $1.4 \times 10^{-2}$ \\
\hline 10 & $5.0 \times 10^{-3}$ & $7.0 \times 10^{-3}$ \\
\hline 12 & $10.1 \times 10^{-4}$ & $19.9 \times 10^{-4}$ \\
\hline 14 & $1.98 \times 10^{-4}$ & $4.23 \times 10^{-4}$ \\
\hline 16 & $29.13 \times 10^{-5}$ & $6.75 \times 10^{-5}$ \\
\hline 18 & $4.15 \times 10^{-6}$ & $8.24 \times 10^{-6}$ \\
\hline $20 \mathrm{~dB}$ & $6.30 \times 10^{-7}$ & $1.0 \times 10^{-6}$ \\
\hline
\end{tabular}

Table III compares the Bit error rate of cyclotomic lattice based open loop system and the proposed cyclotomic lattice based closed loop system at different values of Bit error ratio. It is observed that at BER of value $10^{-3}$, the proposed closed loop system achieves a gain of $0.8 \mathrm{~dB}$.

Table III Bit Error Rate Vs Signal to Noise Ratio

\begin{tabular}{|l|l|l|}
\hline BER & $\begin{array}{l}\text { SNR in dB } \\
\text { (Closed Loop) }\end{array}$ & $\begin{array}{l}\text { SNR in dB (Open } \\
\text { Loop) }\end{array}$ \\
\hline $10^{-1}$ & 0 & 0 \\
\hline $10^{-2}$ & 8.00 & 9.00 \\
\hline $10^{-3}$ & 11.99 & 12.79 \\
\hline $10^{-4}$ & 12.74 & 15.65 \\
\hline $10^{-5}$ & 17.14 & 17.82 \\
\hline
\end{tabular}

Fig.4 compares the symbol error performance of cyclotomic lattice based open loop system and the proposed cyclotomic lattice based closed loop system. It has been observed that the proposed scheme outperforms the conventional scheme.

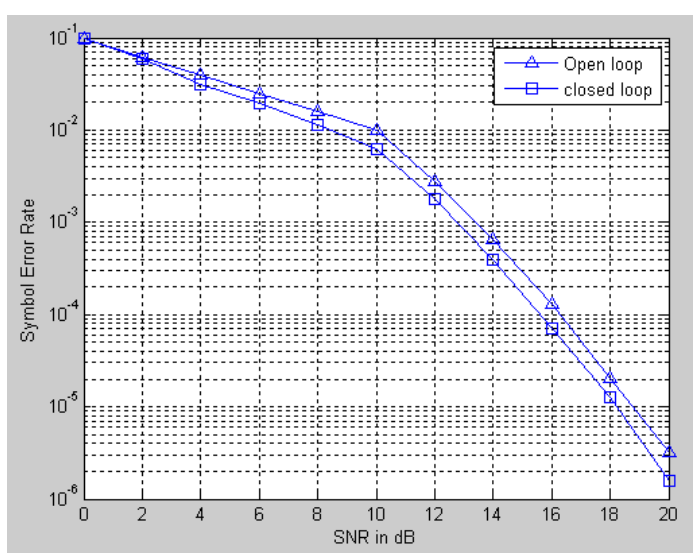

Fig. 4 Symbol error rate versus Signal to noise ratio for lattice based open and closed loop QOSTBC

Published By:

Blue Eyes Intelligence Engineering \& Sciences Publication 
Table IV compares the symbol error rate of cyclotomic lattice based open loop system and the proposed cyclotomic lattice based closed loop system at different values of SNR. It is observed that at $12 \mathrm{~dB}$, the proposed system outperforms the conventional system by $35.71 \%$.

Table IV. Signal to Noise Ratio Vs Symbol Error Rate

\begin{tabular}{|l|l|l|}
\hline SNR in dB & $\begin{array}{l}\text { SER (Closed } \\
\text { Loop) }\end{array}$ & $\begin{array}{l}\text { SER (Open } \\
\text { Loop) }\end{array}$ \\
\hline 0 & $1 \times 10^{-1}$ & $1 \times 10^{-1}$ \\
\hline 2 & $5.9 \times 10^{-2}$ & $6.12 \times 10^{-2}$ \\
\hline 4 & $3.15 \times 10^{-2}$ & $4 \times 10^{-2}$ \\
\hline 6 & $1.95 \times 10^{-2}$ & $2.51 \times 10^{-2}$ \\
\hline 8 & $11.5 \times 10^{-3}$ & $1.6 \times 10^{-2}$ \\
\hline 10 & $6.3 \times 10^{-3}$ & $1 \times 10^{-2}$ \\
\hline 12 & $1.8 \times 10^{-3}$ & $2.8 \times 10^{-3}$ \\
\hline 14 & $4.0 \times 10^{-4}$ & $6.5 \times 10^{-4}$ \\
\hline 16 & $7.11 \times 10^{-5}$ & $13.12 \times 10^{-5}$ \\
\hline 18 & $12.6 \times 10^{-6}$ & $20.2 \times 10^{-6}$ \\
\hline 20 & $1.58 \times 10^{-6}$ & $3.16 \times 10^{-6}$ \\
\hline
\end{tabular}

Table V compares the Symbol error rate of cyclotomic lattice based open loop system and the proposed cyclotomic lattice based closed loop system at different values of Symbol error ratio. It is observed that at SER of value $10^{-3}$, the proposed closed loop system achieves a gain of $0.705 \mathrm{~dB}$.

Table V Symbol Error Rate Vs Signal to Noise Ratio

\begin{tabular}{|l|l|l|}
\hline SER & $\begin{array}{l}\text { SNR in dB } \\
\text { (Closed } \\
\text { Loop) }\end{array}$ & $\begin{array}{l}\text { SNR in dB(Open } \\
\text { Loop) }\end{array}$ \\
\hline $10^{-1}$ & 0 & 0 \\
\hline $10^{-2}$ & 8.5 & 10 \\
\hline $10^{-3}$ & 12.65 & 13.33 \\
\hline $10^{-4}$ & 15.71 & 16.25 \\
\hline $10^{-5}$ & 18.10 & 18.75 \\
\hline
\end{tabular}

\section{V.CONCLUSION}

A novel closed loop QOSTBC system for four transmit antennas based on cyclotomic lattices is proposed in this paper. As every two bits of the input bit stream is mapped to a lattice point, the spectral efficiency of the proposed system is 2 bits per channel use. The channel state information is fedback to the transmitter using 1 bit, which further improves the system performance. From the simulation results, it can be observed that the proposed lattice based closed loop QOSTBC for four transmit antennas outperforms the lattice based open loop QOSTBC system.

\section{REFERENCES}

1. Sundberg, C.,E.W. and Seshadri, “ Digital Cellular Systems for North America", IEEE Globecom, pp.533-7,Dec. 1990. DOI:10.1109/GLOCOM.1990.116568

2. Balaban, $N$ and Salz, J. "Dual Diversity combining and equalization in digital cellular mobile radio ", IEEE Transactions on vehicular Technology, pp.342- 54, May 1994.

DOI: $10.1109 / 25.289415$

3. Winters,J., Salz, J and Gitlin, R.D., " The impact of antenna diversity on the capacity of wireles communication systems", IEEE Transactions oncommunications, pp. 1740-51, April 1994.

DOI: $\quad$ 10.1109/TCOMM.1994.582882
4. Witteneben, A. "A new bandwidth efficient transmit antenna modulation diversity scheme for linear digital modulation", IEEE International Conference on communications (ICC) pp.1630-4, May 1993,.DOI: $\underline{10.1109 / \text { ICC.1993.397560 }}$

5. Seshadri,N. and Winters.J.H. "Two signaling schemes for improvingthe error performance of frequency division duplex (FDD) transmission systems using transmitter antenna diversity" IEEE Vehicular Technology conference, pp.508- 11,May 1993.DOI: $10.1109 /$ VETEC.1993.507522

6. Tarokh, V., Seshdri, N. and calderbank, "Space time codes for high datarate wireless communication: Performance analysis and code construction", IEEE Trans. Inform. Theory, pp.744- 65, Mar. 1998.DOI: 10.1109/18.661517

7. S. M. Alamouti, "A simple transmit diversityTechnique for wireless communications," IEEE J. Select . Areas Commun. vol. 16, pp. 1451- 1458, Oct. 1998.

8. W.Su and X.G. Xia, "Signal constellations for quasi-orthogonal space time block codes with full diversity," IEEE Trans. inform. Theory, vol.50, pp. 2331-2347, Oct. 2004.DOI: 10.1109/TIT.2004.834740

9. Zhu Chen and Moon Ho Lee, "One bit feedback for QuasiOrthogonal Space Time Block Codes Based on Circulant Matrices", IEEE Transactions onWireless Communications,Vol.8, No.7, July 2009. DOI: 10.1109/TWC.2009.080667Wei Liu, Mathini Sellathurai and Jibo Wei, "A Cyclotomic Lattice Based Quasi - Orthogonal STBC for Eight Transmit Antennas

10. ", IEEE Signal Processing Letter, Vol.17, No.4, April 2010 DOI: 10.1109/LSP.2009.2039951

11. Asher Shaji, Anchana C K. Senthil Kumar, R. Amutha, M. Palanivelan, D. Gururaj, S. Richard Jebasingh, M. Anitha Mary, S. Anitha, V. Savitha, R. Priyanka, Amruth Balachandran, H. Adithya, "Receive diversity based rate optimization for improved network lifetime and delay efficiency of wireless body area networks", PLoS One, vol. , no. , pp. 1-20, October 2018. DOI: 10.1371/journal.pone.0206027

12. M. Kanthibathi, R. Amutha, K. Senthil Kumar, "Energy efficient differential cooperative MIMO algorithm for wireless sensor network" Wireless Personal Communications (Springer), vol. 103, no. 4, pp. 2715-2728, December 2018.DOI: 10.1007/s11277-018-5957-1

13. K. Senthil Kumar, R. Amutha, "Energy efficient wireless body area network using receive diversity" Journal of Engineering Science and Technology, vol.13, no. 8, August 2018.

14. K. Senthil Kumar, R. Amutha, "An algorithm for energy efficient cooperative communication in wireless sensor networks", KSII Transactions on Internet and Information Systems, vol. 10, no. 7, pp. 3080-3099, August 2016.DOI: 10.3837/tiis.2016.07.012,ISSN: 1976-7277

15. T.L.K. Sneha Piriya, K. Senthil Kumar, R. Amutha, "Energy Efficient V-MIMO using Turbo Codes in Wireless Sensor Networks" in the Second IEEE International Conference on Computing and Communication Technologies (ICCCT'17), on 24th Feb 2017. DOI: 10.1109/ICCCT2.2017.7972288

16. H.Jafarkhani, "A quasi orthogonal space - Time block code," IEEE Trans. Commun. vol. 49, pp. 1-4, Jan. 2001.

17. K. Senthil Kumar, R. Amutha, "Energy efficient cooperative communication using QOSTBC in wireless sensor networks",International Journal of Advanced Engineering Technology,vol. 7, no. 1, pp. 244-251, March 2016. EISSN: 0976-3945

18. J.H.Conway and N.J.A.Sloane, “ Sphere packing, lattices and groups", $3^{\text {rd }}$ edition, NewYork: Springer - verlag 1998.

19. Genyuan Wang, Huiyong Liao, Haiquan Wang and Xiang- Gen Xia, "Systematic and optimal cyclotomic lattices and diagonal space time block code designs", IEEE Trans. Information Theory, Vol 50, pp. 3348 - 3360, Dec.2004.DOI: 10.1109/TIT.2004.838096

20. G.Wang and X.G.Xia, " On optimal cyclotomicLattices and diagonal / single layer space time block codes", Proc. ISIT 2004, June 27 - July 2, Chicago, USA, 2004.

21. G.Wang and X.G.Xia, "On optimal multi - layer cyclotomic Space -time code designs" IEEE Trans. Inform. Theory, vol. 51, no. 3, pp. 1102-1135, Mar. 2005. 


\section{AUTHORS ProfiLe}

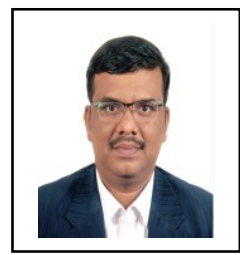

Dr. M. Palanivelan is currently working as an Professor \& Head, Department of ECE, Rajalakshmi Engineering College, Chennai. He received his Ph.D at Anna University in the year 2015 and Master Degree from College of Engineering, Guindy, Anna University, in the year 2001. His research interests include Wireless communications, Information and coding theory, Optical Networks and Multiple antenna wireless communication systems. $\mathrm{He}$ is also a Senior Member of IEEE and also holds professional membership in ACM and Life Member in ISTE.

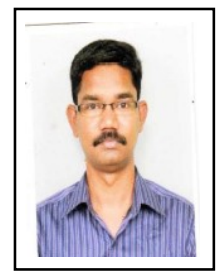

K. Senthil Kumar obtained his Ph.D. at Anna University, Chennai, India in the year 2018. He received his Master Degree from Rajalakshmi Engineering College, Chennai in 2009. In the year 2002, he received his bachelor's degree in Engineering from Bharath Niketan Engineering College, Theni. He is working as an associate professor in the department of ECE, Rajalakshmi Engineering College, India. His research interests include wireless communications, information \& coding theory and MIMO wireless communication systems.

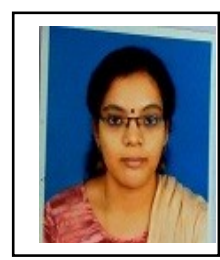

V. Bakyalakshmi obtained her Master Degree in communication systems from Rajalakshmi Engineering College, Chennai in 2016. In the year 2014, she received her bachelor's degree in Electronics and Communication Engineering from Adhiparasakthi College of Engineering, Kalavai. She is working as an assistant professor in the department of ECE, Rajalakshmi Engineering College, India. Her research interests include wireless communication and optical.

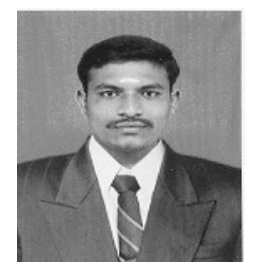

Dr. M.Sivaram, completed his B.E (CSE) at Bharat Niketan Engineering College, Madurai Kamaraj University, Madurai in 2002. He has awarded M.Tech (CSE) degree from National Institute of Technology, Trichy, Tamilnadu in 2007. He Completed Phd degree in Information and Communication Engineering from Anna university ,Chennai in 2014. He has nearly 18 years of experience in Teaching both UG and PG program. He is presently working as an Professor in Department of Information Technology in Lebanese French University, Erbil . His field of interest are Data Mining, Image retrieval, Information retrieval, Data fusion, Image Processing and Artificial intelligence. He has published more than 30 papers in international, National journals and conference.

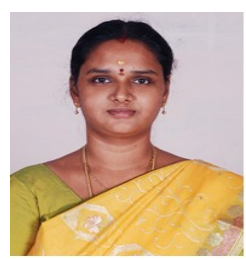

P. Shanmuga Priya received her Master Degree from SASTRA Deemed University, Thanjavur in 2013. She obtained her bachelor's degree in Electronics and Communication Engineering from Kings College of Engineering, Thanjavur. She is working as an assistant professor in the department of ECE, Rajalakshmi Engineering College, India. Her research interests include wireless communication and optical communication. 